\title{
2D ultra-fast MRI of granular dispersion by a liquid jet
}

\author{
Yunan Peng ${ }^{1}$, Andrew J. Sederman ${ }^{1}$, Marco Ramaioli ${ }^{2}$, Eric Hughes ${ }^{3}$, Lynn F. Gladden ${ }^{1}$, and Mick D. Mantle ${ }^{1, *}$ \\ ${ }^{1}$ Department of Chemical Engineering and Biotechnology, University of Cambridge, Pembroke Street, Cambridge CB2 3RA, UK \\ ${ }^{2}$ Department of Chemical and Process Engineering, University of Surrey, Guildford GU2 7XH, UK \\ ${ }^{3}$ Department of Chemistry, Durham University, Lower Mountjoy, South Road, Durham DH1 3LE, UK
}

\begin{abstract}
This paper illustrates the application of ultra-fast magnetic resonance imaging (MRI) as a noninvasive tool to study the dispersion of a dry, static granular bed by the injection of a liquid. Spatial distributions of undispersed grains (poppy seeds) and injected water were independently imaged at submillimetre resolution in 2D with ultra-fast MRI techniques. A liquid jet was observed above the bottom injection orifice, complementing optical imaging. Co-registration of the grains and water images enables the interaction of the static grains and of the liquid jet to be observed for the first time in situ. This visualization of the dispersion process can be used to identify optimal process parameters for a fast and uniform dispersion and to validate quantitatively numerical granular-fluid simulations [1].
\end{abstract}

\section{Introduction}

Whereas the steady fluidization of grains by a fluid has been studied extensively, the transient dispersion of grains initially packed in a heap is not well understood. Dispersion is of interest across many applications: pigments are dispersed into paints, pharmaceuticals powders are dispersed by inhalers, whilst the problem of how to disperse, hydrate [2] and dissolve dehydrated powders is key for many food products [3-5].

Recent advances in magnetic resonance imaging (MRI) hardware and software means it is now possible to study these granular-fluid systems non-invasively and both the solid (granular bed) and liquid may be visualized. This new tool could be vital in investigating optically opaque systems [6,7]. This paper describes how 2D MRI is used to achieve a better understanding of liquid jetting in granular dispersion. This paper reports the first use of MRI to study a three-phase dispersion system. Specifically, we show the interaction of the grains and the liquid during granular dispersion. When a liquid jet is injected upwards into a dry static bed of poppy seeds its decompaction and progressive destruction can be monitored in time using twodimensional magnetic resonance imaging techniques. The results are compared with optical methods and the capabilities and complementarity of these techniques are discussed. In addition, the quantitative measurement of the jetting angle from the MRI data are discussed. Finally, co-registration of 2D liquid and 2D static grain MR images reflects the good reproducibility of the dispersion experiments which are carried out.

\section{Materials and Methods}

In this study, a Perspex dispersion cell with PEEK endcaps, shown in Figure 1, was filled with $4 \mathrm{~g}$ of blue poppy seeds (diameter $\sim 1 \mathrm{~mm}$ ) and then vertically tapped manually 100 times to create a reproducible dry bed packed under gravity. Two distributors made from Scotch-Brite ${ }^{\mathrm{TM}}$ were placed below the injection and exit holes to keep the mass of seeds within the system constant. Water was injected at $200 \mathrm{ml} \mathrm{min}^{-1}$ into the dispersion cell from below, using a constant pressure liquid injection rig relying on compressed air to supply a steady injection pressure and hence liquid flow into the dispersion cell. Excess water leaves the system through the exit hole at the top of the cell at atmospheric pressure, before being evacuated by a peristaltic pump. Both optical imaging and MRI were used to record the dispersion experiments. A high-speed, high resolution digital camera (Photron FASTCAM MC1) was used to obtain optical side-on 512 pixels $\times 512$ pixels images of the dispersion cell at $125 \mathrm{fps}$. The MRI of dispersion experiments were carried out in a wide bore $9.4 \mathrm{~T}$ (400 MHz ${ }^{1} \mathrm{H}$ resonance frequency) superconducting magnet equipped with a Bruker AV spectrometer. A $30 \mathrm{~mm}$ birdcage radio frequency (r.f.) coil was used to excite and detect the ${ }^{1} \mathrm{H}$ nuclei present in the water and the oil of the poppy seeds. Spatial resolution was achieved using a 3-orthogonal axis $(\mathrm{x}, \mathrm{y}, \mathrm{z})$ gradient system capable of delivering a maximum gradient strength of $1.48 \mathrm{~T} \mathrm{~m}^{-1}$.

Two MRI protocols were used to observe the water and grains within the dispersion cell: (i) 2D velocity compensated Fast Low Angle Shot (vc-FLASH) [8,9], which highlights the liquid only; (ii) 2D standard Rapid Acquisition with Relaxation Enhancement (RARE) $[10,11]$, used to image changes to static poppy seeds.

Details of NMR parameters used can be found in Table 1. The 2D vc-FLASH imaging sequence was used to image the liquid component. In this gradient echo sequence, the NMR signal from poppy seeds is two orders of magnitude lower than it is for water due to the

* Corresponding author: mdm@cam.ac.uk 
short $T_{2}{ }^{*}$ of the poppy seeds [12], and thus the liquid phase signal dominates. The central vertical plane of the dispersion cell was studied, allowing the injection hole and its immediate surroundings to be visualised. Velocity compensation was introduced by designing specific gradient waveforms to remove artifacts due to the flow-dependent phase shift of the signal of the ${ }^{1} \mathrm{H}$ nuclei in motion [13].

2D RARE is a spin-echo imaging protocol which utilizes total excitation of the system; however, it suffers heavily from motion artifacts [14]. The inherent nature of the liquid in the system is fast flowing, whilst the granular bed is initially static. For this reason, static poppy seeds in the system can be imaged with the RARE sequence. Additionally, manganese salt $\left(\mathrm{Mn}^{2+}\right)$ doping is used to reduce the $T_{2}$ of the water and hence suppress the water signal [15]. These images enable visualization of the static poppy seed regions during the liquid injection. a)

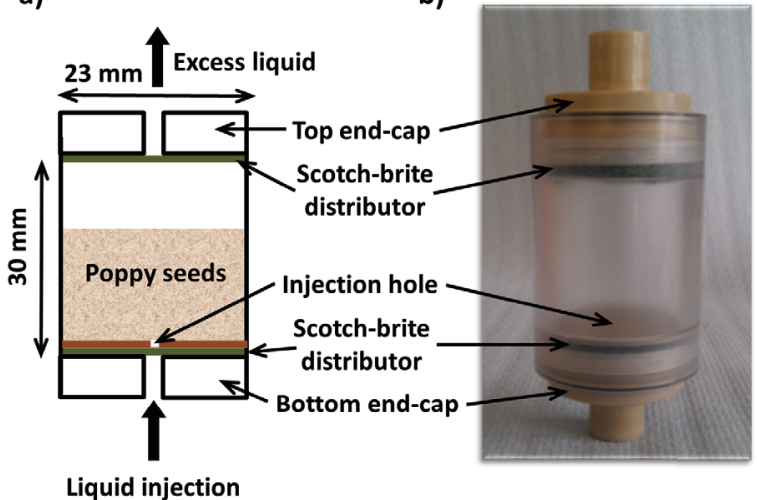

Fig. 1. a) Schematic, and b) photo of dispersion cell used in this study.

Table 1. Parameters for MRI protocols.

\begin{tabular}{|c|c|c|}
\hline & Liquid imaging & Static grain imaging \\
\hline & 2D ve-FLASH & 2D RARE \\
\hline Field-of-view (fov) / mm & $50 \times 50$ & $50 \times 50$ \\
\hline Matrix size / pixel & $64 \times 32$ & $64 \times 64$ \\
\hline Spatial resolution $/ \mu \mathrm{m}$ & $781 \times 1560$ & $781 \times 781$ \\
\hline Slice thickness / mm & 1 & 1 \\
\hline Echo time / ms & 1.3 & 110 \\
\hline Repetition time / ms & 3.0 & 500 \\
\hline Image acquisition time / ms & 100 & 180 \\
\hline Temporal resolution / (fps) & 10 & 2 \\
\hline Injected liquid & \begin{tabular}{|c|}
$2.5 \mathrm{mM} \mathrm{Gd}^{3+}$ solution \\
$\left(\mathrm{T}_{1}=29 \mathrm{~ms}, \mathrm{~T}_{2}=23 \mathrm{~ms}\right)$
\end{tabular} & $\begin{array}{l}15 \mathrm{mM} \mathrm{Mn}^{2+} \text { solution } \\
\left(\mathrm{T}_{1}=7 \mathrm{~ms}, \mathrm{~T}_{2}<1 \mathrm{~ms}\right)\end{array}$ \\
\hline Granular material & \multicolumn{2}{|c|}{ Poppy seeds $\left(\mathrm{T}_{1}=450 \mathrm{~ms}, \mathrm{~T}_{2}=78 \mathrm{~ms}\right)$} \\
\hline
\end{tabular}

\section{Results and Discussion}

\subsection{Optical imaging}

The capture of optical images using a high-speed, highresolution camera allows the dispersion of poppy seeds in the geometry shown in Figure 1 to be appreciated.

Figure 2 shows a time series of images obtained where water is injected at $200 \mathrm{ml} \mathrm{min}^{-1}$ into a $4 \mathrm{~g}$ bed of poppy seeds in the dispersion cell. Initially a dry static bed of poppy seeds can be seen, before decompaction of the poppy seed bed between $t=0 \mathrm{~s}$ and $\mathrm{t}=0.2 \mathrm{~s}$. Water is then flowing through the centre of the packed bed between $\mathrm{t}=0.2 \mathrm{~s}$ and $\mathrm{t}=1.0 \mathrm{~s}$. After $\mathrm{t}=0.6 \mathrm{~s}$, a water layer forms above the bed. This layer of water is turbulent with air bubbles and poppy seeds present and increases in height until it reaches the top of the dispersion cell at around $\mathrm{t}=2.0 \mathrm{~s}$. The system reaches a steady state after $\mathrm{t}=2.0 \mathrm{~s}$ for this dispersion experiment with a turbulent region of water, poppy seeds and air bubbles formed above the remains of the poppy seed bed. The liquid injection was continued for 5 minutes, but the system did not evolve significantly after $2.5 \mathrm{~s}$.
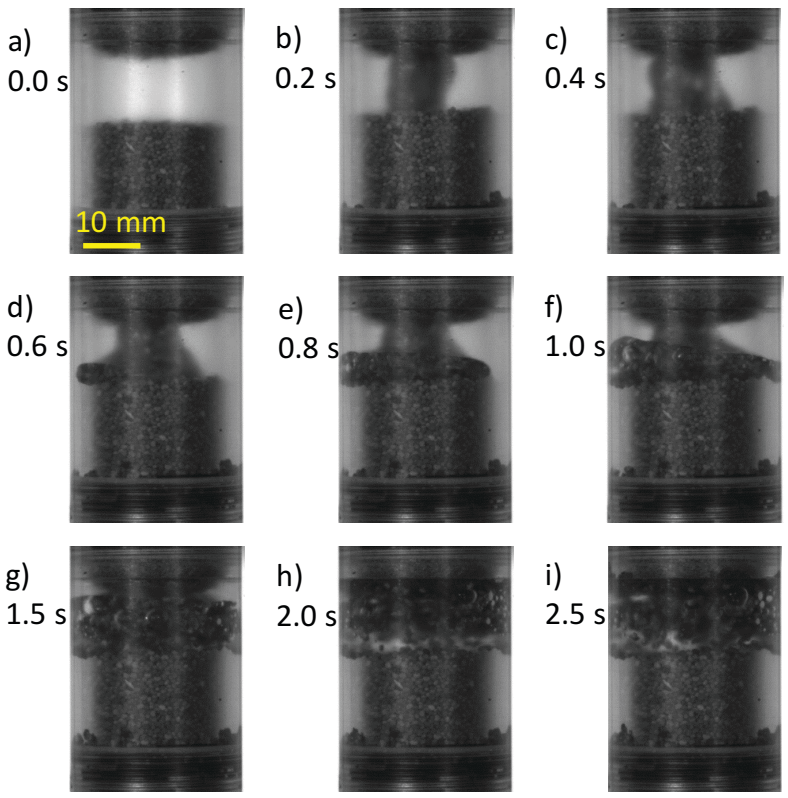

Fig. 2. A selection of optical images recorded at 512 pixels $\times$ 512 pixels during the dispersion of poppy seeds by water. At $0.0 \mathrm{~s}$ water enters the bottom of the cell. Note the dispersion cell is of constant radius, however the difference in refractive index between water and air gives the illusion that wetted areas of the dispersion cell appears wider.

\subsection{Liquid imaging: 2D vc-FLASH}

a)

b)

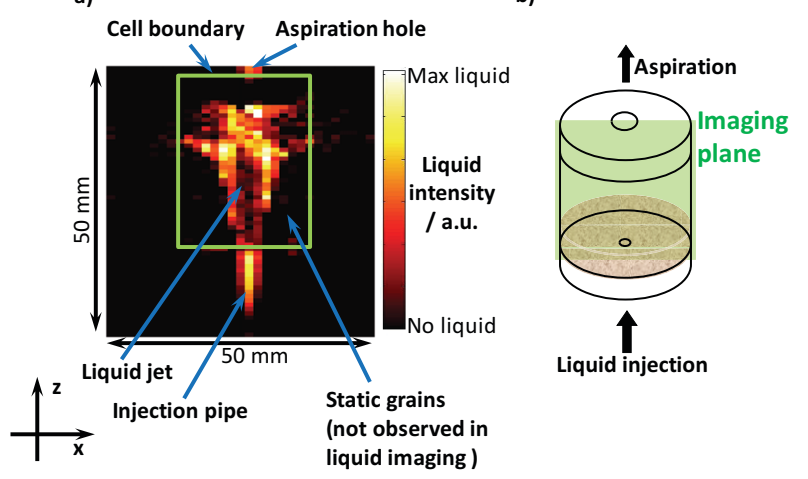

Fig. 3. a) A typical snapshot of 2D selective liquid imaging of the central vertical slice of a dispersion experiment. The jet can be seen rising from the bottom of the cell (green boundary) in a plume. b) Schematic of the vertical imaging plane.

Figure 3 a) shows a steady state 2D vc-FLASH image $4.0 \mathrm{~s}$ after the start of liquid injection into a bed of poppy seeds in the central vertical slice through the dispersion 
cell depicted in Figure 3 b). A well-developed steady state liquid jet is shown. The dark areas in the $2 \mathrm{D}$ vcFLASH map correspond to areas without liquid signal; the two large areas around the bottom circumference of the cell are the regions of stagnant poppy seeds, which are not seen in liquid imaging due to its much lower signal than water. It is important to note that although a scale bar with two limits is given, reduced pixel intensity is also likely due to signal loss as a result of the high shear present within a voxel, especially with the high liquid flow rates involved. For example, at the central core of the liquid jet shows low signal, which is likely due to the water moving too fast to be imaged rather than lack of water. The superficial velocity for water flowing through the $1 \mathrm{~mm}$ injection hole at $200 \mathrm{ml} \mathrm{min}^{-1}$ is $1.1 \mathrm{~m} \mathrm{~s}^{-1}$. Therefore, during the $1.3 \mathrm{~ms}$ echo time, a voxel of water will move on average $1.43 \mathrm{~mm}$. This does not compare favourably with the spatial resolution of $781 \mu \mathrm{m}$ in the vertical direction and is a reason for signal loss.

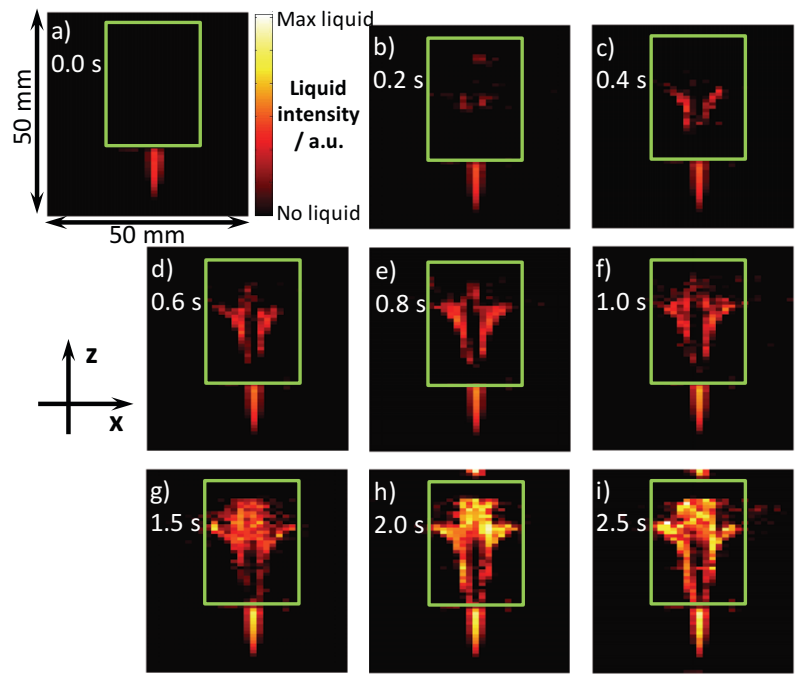

Fig. 4. A selection of liquid-imaging snapshots of the central vertical slice acquired using 2D vc-FLASH for a dispersion experiment with water jetted into a granular bed of poppy seeds. Temporal resolution: $10 \mathrm{fps}$. Spatial resolution: $0.781 \mathrm{~mm} \times$ $1.560 \mathrm{~mm}$. The time is normalized such that $\mathrm{t}=0 \mathrm{~s}$ corresponds to water reaching the bottom of the dispersion cell.

Figure 4 shows a time series of 2D image vcFLASH snapshots during the approach to steady state. where water is injected from the bottom into the poppy seeds bed. The water injected into the dispersion cell was observed to penetrate through the middle of the granular bed. The water can already be seen to have penetrated completely through the poppy seeds by $\mathrm{t}=0.6 \mathrm{~s}$ as signal is seen from the top of the dispersion cell. The initial liquid jet then forms from the top-down between $t=0.4 \mathrm{~s}$ and $\mathrm{t}=0.8 \mathrm{~s}$. At this point, a steady jet has formed and this stabilizes over the next second as the intensity of the jet increases; the system is then defined to have reached steady state at $\mathrm{t}=2.0 \mathrm{~s}$ as very little change is seen in the jet thereafter. The increase in signal intensity is because of lower velocity attenuation at steady state compared with in the initial dry dispersion cell. As more water is injected into the dispersion cell, the distribution area of liquid jet widens, therefore the vertical velocity profile of the jet reaches a lower maximum.

The results show the symmetry of the jet is as expected at steady state, although it was unable to provide further insight into determining how the initial changes in the dispersion cell are taking place, due to the much shorter timescale of this phenomenon, i.e. of the order of hundreds of microseconds.

The 2D spatial maps have allowed the internal water flow to be directly visualized non-invasively and in situ. When injecting a liquid in dry grains, after the jet has broken through the granular bed and reached the top of the cell, the liquid is not directly aspirated through the exit hole, but rather cascades down the side of the dispersion cell. It then builds up above the regions where grains are static and it wicks in the grains downwards. The liquid is therefore partly above the dry granular regions, as shown in Figure 4 at $\mathrm{t}=2.0 \mathrm{~s}$ and $2.5 \mathrm{~s}$.

\subsection{Grain imaging: 2D RARE}

a)

Cell boundary

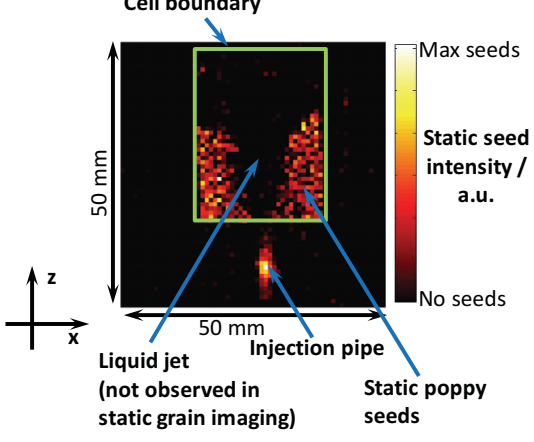

b)

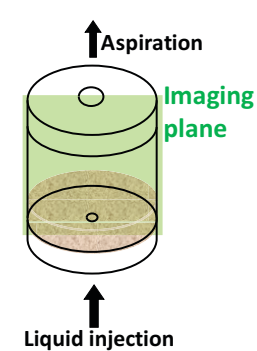

Fig. 5. a) A typical 2D RARE snapshot of the central vertical slice during a dispersion experiment. The static grains are pushed towards the outer edge of the bottom of the cell. Liquid is not visible, except for an artefact below the dispersion cell, at the injection pipe. b) Schematic of the vertical imaging plane.

Two dimensional RARE was used to produce 2D spatial maps of the static poppy seeds in the same central vertical plane as shown in Figure $3 \mathrm{~b}$ ) for liquid imaging with 2D vc-FLASH. Figure 5 a) shows the corresponding 2D RARE image to the liquid spatial map in Figure $3 \mathrm{a}$ ). The image is taken $\mathrm{t}=4.0 \mathrm{~s}$ after the start of liquid injection into the bed of poppy seeds in the central vertical slice through the dispersion cell depicted in Figure $5 \mathrm{~b}$ ). With the liquid signal suppressed, the signal observed arises from the poppy seed oil in static, or stagnant, poppy seeds. When taken together with Figure 3 a), it can be concluded a liquid jet at the centre of the dispersion cell is observed with a ring of stagnant seeds around the circumference at the bottom of the cell. The fluid intensity is lower at the bottom of the cell, where the stagnant seeds are imbibed with some fluid. Water can be observed on top of the regions of static seeds. Figure 6 shows a time series of snapshots in an equivalent experiment to that shown in Figures 2 and 4. Here, a growing vacant region can be seen in the centre of the granular bed, while static grains progressively disappear. The co-registration presented in the following section helps interpret this pattern more clearly. 


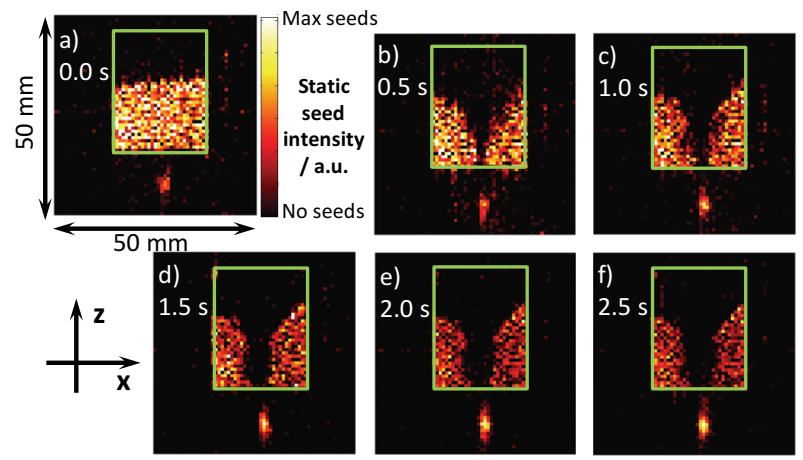

Fig. 6. A selection of grain-imaging snapshots of the central vertical slice acquired using 2D RARE. Dispersion of a granular bed of poppy seeds with injected water. Temporal / Spatial resolution: $0.781 \mathrm{~mm} \times 1.560 \mathrm{~mm} ; 10 \mathrm{fps}$.

\subsection{Co-registration: 2D liquid and grain imaging}

With the two dimensional spatial maps of liquid and powder imaging, it is possible to study the liquid-grain interactions within an optically opaque system. The coregistration of these spatial maps has proven the reproducibility of the dispersion experiments carried out, with the static bed-liquid jet interface being directly visualised.

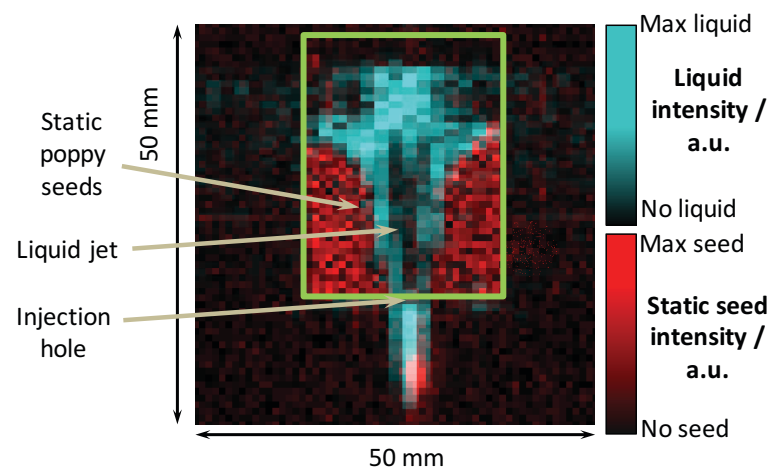

Fig. 7. Co-registration of 2D liquid and grain spatial maps, obtained after $2.5 \mathrm{~s}$ of water injection at $200 \mathrm{ml} \mathrm{min}^{-1}$ into $4 \mathrm{~g}$ of poppy seeds. The water jet is shown in cyan, the stationary poppy seeds in red and the dispersion cell is outlined in green.

A co-registration image of $2 \mathrm{D}$ liquid and static grain spatial maps is shown in Figure 7. The cyan channel represents the water jet and the red channel represents the static grains in the system. A ring of stagnant poppy seeds is seen around the outer edge at the bottom of the dispersion cell. Although the two spatial maps are obtained using two different methods and acquired during separate experiments, the liquid jetstatic poppy seed boundary overlaps well. This image shows both the injection of water into the system and the static granular bed dispersed and eroded away. It directly reflects simulations of the central vertical plane of the dispersion cell [1], where a low porosity jet is immediately surrounded by high porosity regions towards the circumference of the bottom of the dispersion cell. This data complement optical methods to provide a fundamental understanding of the process taking place during powder dispersion.
This powerful MRI technique could also be used to extend the physical understanding of the formation of air jets in a liquid saturated granular medium $[16,17]$ or of wicking of a dry powder [18].

\section{Conclusions}

The dispersion of a dry static granular bed by liquid injection was investigated using ultra-fast MRI. Whilst optical methods are limited by the opaqueness of these 3D dispersion set-ups, sub-millimetre resolution images of two phases during dispersion were achieved using ultra-fast MRI protocols. MRI can characterise quantitatively the dispersion due to liquid jetting and how the surrounding regions of static poppy seeds evolve over time. Good reproducibility for dispersion of poppy seeds was achieved. This enables the coregistration of the liquid and static grain spatial maps, enabling in turn granular dispersion to be studied in situ.

The authors are grateful to Nestlé Research for financial support and to EPSRC Loan Pool for contributing towards the optical imaging of dispersion.

\section{References}

1. M. Robinson, M. Ramaioli, S. Luding, AIP Conf. Proc. Powder and Grains 2013, 1542, 1122 (2013)

2. J. Dupas, E. Verneuil, M. Ramaioli, L. Forny, L. Talini, F. Lequeux, Langmuir 29, 40 (2013)

3. P. Schuck, A. Davenel, F. Mariette, V. Briard, S. Méjean, M. Piot, Int. Dairy J. 12, 51 (2002)

4. L. Forny, A. Marabi, S. Palzer, Powder Technol. 206, 72 (2011)

5. J. Dupas, L. Forny, M. Ramaioli, Journal of Colloid and Interface Science 448, 51 (2015)

6. M.D. Mantle, A.J. Sederman, Prog. Nucl. Magn. Reson. Spectrosc. 43, 3 (2003)

7. C.R. Müller, D.J. Holland, A.J. Sederman, M.D. Mantle, L.F. Gladden, J.F. Davidson, Powder Technol. 183, 53 (2008)

8. A. Haase, J. Frahm, D. Matthaei, W. Hanicke, K.D. Merboldt, J. Magn. Reson. 67, 258 (1986)

9. J. Frahm, A. Haase, D. Matthaei, J. Comput. Assist. Tomogr. 10, 363 (1986)

10. J. Hennig, A. Nauerth, H. Friedburg, Magn. Reson. Med. 3, 823 (1986)

11. J. Hennig, H. Friedburg, Magn. Reson. Imaging 6, 391 (1988)

12. T.M. Nguyễn, A.J. Sederman, M.D. Mantle, L.F Gladden, Phys. Rev. E 84 (2011)

13. J.M. Pope, S. Yao, Concepts Magn. Reson. 5, 281 (1993)

14. B. Madore, R.M. Henkelman, J. Magn. Reson. Imaging 4, 577 (1994)

15. A.P. Koretsky, A.C. Silva, NMR Biomed. 17, 527 (2004)

16. M. Jankov, G. Løvoll, H.A. Knudsen, K.J. Måløy, R. Planet, R. Toussaint, E.G. Flekkøy, Transport Porous Med. 84, 569 (2010)

17. B. Sandnes, E.G. Flekkøy, H.A. Knudsen, K.J. Måløy, H. See, Nat. Commun. 2, 288 (2011)

18. P. S. Raux, H. Cockenpot, M. Ramaioli, D. Quéré, C. Clanet, Langmuir, 29 11, (2013) 